MODELING, IDENTIFICATION AND CONTROL, 1994, VOL. 15, NO. 3, 153-159

doi:10.4173/mic.1994.3.4

\title{
System identification in a MIC perspective
}

\author{
LENNART LJUNG $\dagger$
}

Keywords: Identification; modeling; model errors; disturbance models.

The paper describes some subjective aspects on some current research topics in system identification. A 'classical' standpoint is taken regarding reduced complexity models. The need for specific tools for 'semi-physical modeling' is also pointed out, and a discussion on different disturbance models is also included.

\section{Modeling, identification and control}

The role of mathematics to help people organize their thinking is one of the more fascinating themes in the human culture. The more philosophical aspects of this will be left aside in this contribution, but an important fact is that mathematically based analysis and synthesis plays a rapidly increasing role for engineering problems. This means of course that mathematical models of engineering objects become crucial.

Ever since Maxwell's and Vyshnegradski's analysis of basic control solutions, mathematical models - mostly in terms of differential equations - have been a main ingredient in control engineering. This has of course been more pronounced after the birth of 'modern control theory' around 1960.

The control community has taken an active interest in developing techniques to estimate models of dynamical systems from observed input and output data and has termed that process 'System Identification'. The traditional techniques for this have close relationships to statistical methods, and in particular to Time Series Analysis, but some aspects of this problem were first developed in the control community. System Identification has for the past 25 years been a standard topic in control conferences and in the control literature. It is interesting to see how the amount of papers and conference sessions on identification has rapidly increased over the past two years, after steadily dropping during the $1980 \mathrm{~s}$.

Physical modeling is in a sense at the other end of the spectrum for constructing mathematical models. Modeling means that known basic physical laws about the object are put together and organized into a form, a model, that is suitable for simulation, analysis and/or synthesis. While this process is as important for the model construction as identification, it has for some reason never played the same role in the control research community. In practical applications the processes of modeling and identification go hand in hand, and tools that integrate the techniques are highly desirable. More about that in Section 3.

The use of a model can be very versatile, and analysis and synthesis for control purposes is only one aspect. It has throughout the history of System Identification been stressed that the model must be developed with the intended use in mind. However, only recently there has emerged a specific 'Identification for Control' literature.

It is my purpose with this contribution to give a perspective on Identification, its current trends and future needs. I will do that in the following two sections by first

Received 9 February 1994

† Department of Electrical Engineering, Linköping University, S-581 83 Linköping, Sweden. 
focusing on Identification for Control and then on the interplay between Modeling and Identification. In this way the three main topics of MIC will be woven together. I should stress that the perspective is subjective and that it reflects my own current interests, and in no way can be read as a survey of what is happening in the field.

\section{Identification for control}

To link the desired properties of the sought model to its intended use is an obvious thought. Adaptive control can be seen as the ultimate process of this kind, where the (recursively) identified model is immediately used for design, or tuning, of the regulator, and then discarded. Adaptive control using a model in this way was also suggested very early (Kalman 1958).

Off-line procedures for identifying models, that are specifically tailored to be good for control design (often using a specified control criterion) are however a quite recent research topic. See Gevers (1993) for a survey. We shall review a few aspects around this topic here.

\subsection{Bias and variance errors}

There are two (and only two!) reasons why identification of models may be difficult.

- The observed data are not exactly reproducible (due to 'unmeasured signals' that also affect the measurements).

- The observed data do not cover all situations in which the model is to be used. We thus need 'interpolation and extrapolation' rules between the measurements in the observation space.

Therefore, all estimated models are subject to errors. These are correspondingly of two kinds:

- Variance errors (or random errors), which stem from the fact that the observations are not exactly reproducible. These errors are often described in a stochastic framework.

- Bias errors (or systematic errors). These originate from the fact that the underlying model structure ('the interpolation and extrapolation rules') is inappropriate, typically too simple.

In the use of the model, it is of course the total error (=the sum of bias and random errors) that matters, but the distinction of its components is quite important to understand how to affect it. It is, for example, typically so, that the random error increases and the bias error decreases as the model structure flexibility ('model order') increases.

\subsection{Minimizing the total error}

We may conceptually write the total error as a sum of the bias and random errors:

$$
V_{l}(d)=V_{b}(d)+V_{r}(d)
$$

Here $d$ is a real (or integer) number that measures the flexibility of the underlying model structure, such as the number of free parameters. Suppose that, as $d$ increases

- $V_{b}(d)$ decreases

- $V_{r}(d)$ increases

- $V_{r}(d) * V_{b}(d)$ decreases 
These are all typical properties of the random and bias errors. Then it follows that for the value $d^{*}$ that minimizes the total error $V_{t}(d)$ we have

$$
V_{b}\left(d^{*}\right) \leqslant V_{r}\left(d^{*}\right)
$$

The conclusion is thus that model complexity that minimizes the total error of some aspect of a model, automatically is such that the bias eiror (of that aspect) is dominated by the random error. See Guo and Ljung (1994) for a proof and a discussion around this.

\subsection{Model validation}

Modcl validation is the process of confronting the estimated model with various facts about the system, and see if these facts may falsify it. Most often the 'facts' are another observed data set-the validation data. Traditional model validation tests include residual tests, in which the left-overs from the model are tested for independence (non-correlation) with past inputs.

Conceptually, the model validation amounts to checking whether the bias error is larger than the random error. A model whose bias error cannot be established without much doubt (it is 'concealed' in the random error) is thus not falsified. This obviously links well with seeking the model that has the smallest total error, according to what was said above.

In Guo and Ljung (1994) this conceptual view of model validation is substantiated for linear models.

\subsection{Reduced complexity models: identification for control}

It is clear that we cannot and should not seek a model whose bias error is zero. One reason is that such a model structure would be very complex, if at all feasible. Another, and more pragmatic, reason is that such a model would not minimize the total error. There can be no practical reason for accepting a model that is more complex and at the same time less accurate than another.

In this sense, all estimated models in practical use are of reduced complexity.

However, there might be reason to use models that are simpler than the one that minimizes the total error, basically for the ease of the design process. For such a model typically the bias error is the dominating contribution to the total error. Consequently, such models would normally be falsified during model validation. These are then reduced complexity models.

If the bias error dominates the total error, it is of importance to know that it can be 'shaped'. That is, within a given model structure, some system properties can be given more attention than others. (For linear models, we may think of approximations of the frequency function that focus on certain frequency ranges.) This shaping can be achieved both by the experimental conditions at hand when the data are collected and by various data polishing tricks, such as prefiltering. Various ways of affecting the bias error (as well as the random error) are summarized, for example in Ljung (1987).

There is a tremendous current interest in estimating and dealing with reduced complexity models, assessing their total error, and finding out how to shape their bias, so that controllers computed from them give the best possible closed loop behaviour. This is one of the most active subfields in System Identification today. See the special issue (Kosut, Goodwin and Polis 1992) with many interesting contributions. 


\subsection{My summary}

I have, after various detours, come to a conservative and classical standpoint about reduced complexity models, identification for control, bias estimation, and the like:

(1) Build a model that passes a model validation test (focusing on the aspects of the model that are relevant for its purpose. For a linear model, this might mean that past inputs are uncorrelated with the residuals, filtered through a filter with passband equal to the frequency range where a good model is needed).

(2) If this validated model is too complex to be suitable for its purpose (like control design), reduce it (again focusing on the important aspects).

(3) To assess the total error of the resulting, reduced complexity, model, first estimate the random error of the validated model. Its bias error is then also dominated by this error, according to what was said above. Finally add to the error the discrepancy between the validated and the reduced models.

The reasons for this procedure are as follows:

- In practical experience, unfalsified models of moderate complexity are often obtained.

- The simplest unfalsified model is likely to have the smallest total error. There seems to be no reason not to compute such a model, even if even simpler ones are desired/needed for the control design.

- If we cannot achieve an unfalsified model in the class of model structures we are working with (for example due to non-linear system properties and linear models), then it is not likely that we can assess the total error of any reduced order model either.

When we are dealing with building models from one given experiment in an off-line fashion, I think it is quite obvious that one must first look for the simplest unfalsified model. This is of course entirely in line with the classical modeling point of view, but this starting point has been somewhat overshadowed in the recent literature.

A somewhat different situation is at hand in several approaches for 'identification for control' which deal with iterative schemes, where new experiments are designed, based on the outcome of previous ones. I think that the basic attitude should still be to look for a model than cannot be falsified using data from all the experiments, and then, if necessary, reduce that. If an unfalsified model cannot be found it means that we have no confidence in the mechanisms that generate the output under varying experimental conditions. It might then be tempting to use the model that describes the system best under conditions that resemble the intended closed loop properties. There may be some risks associated with that, though.

\section{Modeling and identification}

The type of model structures used in estimation can be divided into two classes:

(1) Black boxes. By black boxes are meant families of models (often linear ones), whose parameters do not have physical significance, but where the objective is to find a good model that fits the observed data. For linear models we can think of black boxes as parameterizations of the frequency functions in terms of various function expansions. For non-linear models, Artificial Neural Networks represent the type of black-box structures that have received most of the recent interest. 
(2) Physically parameterized models. These are the results of more or less laborious modeling, where all physical insight about the behaviour of the process is condensed into a model, usually in state space form, which contains both known and unknown parameters. The unknown parameters describe the model structure, and they typically have a physical significance of their own, such as unknown physical constants, and so on.

From an estimation/identification point of view there is no difference between the two approaches; they just differ in the parameterization of the predictors, and all estimation theory (convergence, consistency, accuracy) remains the same. This means, in my mind, that it is not suitable to reserve the term identification for the black-box case only, which is sometimes done.

The second approach really involves full fledged modeling, and it could be a very time consuming task to come up with a good structure. Also, it is often quite difficult to keep the number of parameters to be estimated down to a reasonable figure. While good software tools for physical modeling now are becoming available, for example, Dymola (Elmquist 1993), it is an important task for the near future to develop software tools that closely integrate the modeling and identification aspects. One attempt in this direction is described in Nagy and Ljung (1991) where bond-graph modeling is integrated with identification of state space models, but much more needs to be done in this area.

\subsection{Semi-physical modeling}

Between the two 'extremes' on the scale of design of a model structure there is a zone where considerable and important physical insight is used in the identification process, but not to the extent that a formal physically parameterized model is constructed. We shall here call this zone Semi-physical modeling. The approach is by no means new, but I strongly believe that it is time to formalize aspects of it and develop methods and software tools that will assist in its application.

By semi-physical modeling I mean the process of taking physical insight about the behaviour of the system into account, and using that insight to come up.with suitable non-linear transformations of the raw measurements so that the new transformed variables the new inputs and outputs - stand a better chance to describe the true systern when they are subjected to standard model structures (typically linear in the new variables). This could be a simple matter of using the squared voltage rather than the voltage itself as an input, if we know that the consumed power is the basic stimulus in the system. It could also be a matter of much more complicated transformations, that are suggested based on computer-algebraic treatment of basic physical laws, and tested en masse against measured data.

This type of approach, 'between' black-box identification and physical modeling, is in most practical cases the most promising one. While it no doubt is frequently used in many thoughtful applications of identification, it is also true that many failures in this area can be blamed on a missing, essential (and in retrospect, trivial) non-linear transformation. The need for developing tools for the approach is obvious.

This will require synthesis of methods from different areas. Based on physical considerations the modeler is often able to write down some fundamental equations, which are believed to approximately describe the plant in question. Apart from the measured signals, such equations typically also include imaginable nonmeasured/non-measurable signals, say $x(t)$. These signals need to be eliminated using 
some symbolic equation solver. If the original physical equations are differentialalgebraic, then there is a considerable support for this both regarding theory, algorithms and software. Commutative algebra and Gröbner bases are examples of such theoretical tools. The result of the operation is typically a quite complicated rearrangement of the original equations. In the semi-physical modeling approach one is less concerned about the exact expression that results from the manipulations. It is more interesting to look at the character of the non-linearly transformed measured variables that appear in these expressions. These are candidates to be regressors in a linear regression model of the plant. There are often so many, though, that they need to be efficiently sorted and screened, both with regard to their internal relationships and to their proven correlation with the process signals to be modeled. The latter involves a number of statistical tests, closely related to linear regression modeling, see for example Draper and Smith (1981). Some ideas along these lines are presented in Lindskog and Ljung (1994).

\section{Probabilistic and unknown-but-bounded disturbances}

Of course, there may be good reasons for questioning the traditional description of disturbances and noises as realizations of random processes. In fact, over the past few years, there has been a considerable interest in the identification literature in approaches that reject probabilistic descriptions of the disturbances. This includes both the areas of 'unknown-but-bounded disturbances' (Schweppe 1968) 'Set membership identification' (Fogel 1979), 'Optimal algorithms' (Milanese and Tempo 1985) and 'Worst case analysis' or 'Robust identifiability' (Mäkilä and Partington 1991).

While these approaches and analytic results have given most valuable insights, it is also true that the results are characterized by being very conservative. The worst case bounds are often unrealistically large compared to what practical experience shows. In a stochastic setting one would say that the noise sequences that would give the worst case bounds are 'extremely unlikely'. Such results are nevertheless of interest. However, one should be careful, I think, to design the actual estimation algorithms based on these bounds, neglecting their 'typical' performance for real data.

One basic concept that $\mathbf{I}$ think should be addressed in some way, even in the unknown-but-bounded noise case, is that of 'independence' between disturbances and inputs. This notion is really the only thing that matters in the probabilistic approach to identification. In my mind, a 'disturbance' that is not independent of the inputs is a model error and not a disturbance (Hjalmarsson and Ljung 1993).

Another reason to work with errors that do not depend on the input is closely linked with model validation. In a somewhat unfair summary one could say that the non-probabilistic, validated model message to the user is as follows: Here is a model. On past measured data it has never produced an error larger than so-and-so. The implication is of course that one could then expect that it won't produce any larger errors in the future either. The parallel probabilistic statement is as follows: Here is a model. On past data it has produced errors with an (empirical) distribution of so-and-so. Again the implication is supposed to be that this error distribution should be representative for the future. However, a probabilistically validated model comes with the following important additional statement: and there is no evidence in past data that the errors in any way originate from the inputs. Notice that it is this extra statement that gives us confidence to use the model for other input characteristics than those of the past data! 


\section{Some conclusions}

The identification area is as active as ever as a research and application field. For its links to control, I have pointed to some, perhaps controversial, thoughts about how to deal with reduced order models. I have also pointed out the strong need for linking identification and modeling closer. In particular tools and methods for dealing with physical insight going through a complete modeling stage are in great need. For nonprobabilistic disturbance models, I argued that anyway some independence measure is required.

I would also add, that for the future there is a strong need to move on to model concepts (and then modeling and identification tools) that are capable of better describing the real world objects. These often require elements both of conventional differential/difference equation models and of capabilities to describe various modes and the logics how the modes of operation relate to each other.

Finally I think that we must realize that large real-time databases, stored in primary memory, and thus very fast, are becoming standard. This will no doubt have a substantial influence on the model concept and how we deal with models and model based control and supervision.

\section{REFERENCES}

Draper, N. R. and Smith, H. (1981). Applied Regression Analysis, 2nd ed. (Wiley, New York). ELMQUIST, H. (1993). Object-oriented modeling and automatic formula manipulation in Dymola. In Proc. SIMS'93, Scandinavian Simulation Society, Kongsberg, Norway, June, 1993, pp. 97-105.

FOGEL, E. (1979). System identification via membership set constraints with energy constrained noise. IEEE Trans. on Automatic Control, 24, 615-622.

Gevers, M. (1993). Towards a joint design of identification and control? In H. L. Trentelman and J. C. Willems, eds, Essays on control: Perspectives in the theory and its applications, ECC'93 Groningen.

Guo, L. and LJung, L. (1994). The role of model validation for assessing the size of the unmodelled dynamics. Technical Report LiTH-ISY-I, Department of Electrical Engineering.

Hualmarsson, H. and LJUNG, L. (1993). A discussion of 'unknown-but-bounded' disturbances in system identification. In Proc. 32nd IEEEE Conf. on Decision and Control, San Antonio, Texas, 1993.

Kalman, R. E. (1958). Design of a self-optimizing control system. Transaction ASME, Journal of Basic Engineering, 80, 468-478.

Kosut, R. L., Goodwin, G. C. and Polis, M. P. (Eds). (1992). Special Issue on System Identification for Robust Control Design, IEEE Trans. Automatic Control, 37.

Lindskog, P. and LJUng, L. (1994). Tools for semi-physical modelling. In Proc. IF AC Symposium on Identification and System Parameter Estimation, Copenhagen, Denmark.

LJUNG, L. (1987). System Identification-Theory for the User (Prentice-Hall, Englewood Cliffs, N.J.).

Mäkilä, P. and Partington, J. (1991). Robust approximation and identification in $h_{\infty}$. Proc. American Control Conference, pp. 70-76.

Milanese, M. and TeMPO, R. (1985). Optimal algorithms for robust estimation and prediction. IEEE Trans. Automatic Control, 30, 730-738.

NAGY, P. A. J. and LJUNG, L. (1991). System identification using bond graphs. In Proceedings of the European Control Conferences, 3, pp. 2564-2569, Grenoble.

SCIIWEPPE, F. C. (1968). Recursive state estimation - unknown but bounded errors and system inputs. IEEE Trans. on Automatic Control, 37, 22-28. 